\title{
Successful Aging: Multiple Trajectories and Population Heterogeneity
}

\author{
Fengyan Tang \\ Correspondence: Fengyan Tang, School of Social Work, 2217C Cathedral of Learning, University of Pittsburgh, \\ Pittsburgh, PA, 15260, USA. E-mail: fet7@ pitt.edu
}

Received: March 20, 2014

Accepted: April 16, 2014

Available online: April 23, 2014

doi:10.11114/ijsss.v2i3.372

URL: http://dx.doi.org/10.11114/ijsss.v2i3.372

\begin{abstract}
Following Rowe and Kahn's successful aging model, this study identified successful aging as a distinctive aging trajectory and examined gender differences in the aging process. Using the Health and Retirement Study data (2000-2008), this study applied group-based trajectory analysis to identify multiple aging trajectories in a sample of older Americans aged 65 and over $(N=9,226)$. Six dimensions were analyzed in the multi-trajectory model: chronic disease, physical functional limitation, disability, depressive symptom, cognitive functioning, and active engagement with life. Three aging trajectory groups were identified: successful aging, usual aging, and pathological aging in both samples of men and women. About one third of women and 40 percent of men fell into the successful aging trajectory. Women were more likely than men to experience functional limitations and disability over time, thus less likely to age successfully. Younger age and higher levels of education and income were related to the likelihood of successful aging. Findings provide support for a multidimensional model of successful aging that emphasizes the heterogeneity in physical, cognitive, and social functioning among the older population. Future research need incorporate subjective assessments of successful aging, examine the potential transitions among different aging trajectories, and assess the impacts of health behavior and resource variables on the aging outcomes.
\end{abstract}

Keywords: successful aging, aging trajectory, gender difference

\section{Introduction}

Successful aging has been a subject of increasing public interests and research efforts since Rowe and Kahn proposed this aging model in the late 1980s. Although there is no consensus on how to define and measure successful aging, the three-component model developed by Rowe and Kahn (1997) has been widely accepted. The model provides a multidimensional conceptualization of successful aging, including low risk of disease and disability, high physical and cognitive functional capacity, and active engagement with life (Rowe \& Kahn, 1997). Based on these dimensions, at least three distinct pathways in the aging process could be postulated: pathologic, usual, and successful aging. Older adults with specific diseases or disability may experience pathologic aging; their lives have been affected by the diseases since adulthood or even earlier. In contrast, older adults without major diseases or disability may experience either usual aging (non-pathologic but high risk for specific diseases) or successful aging (low risk and high function) (Rowe \& Kahn, 1987; 1997).

Previous research focused on the prevalence estimate of successful aging in the United States. Surprisingly, a huge difference existed in the prevalence estimates from the studies of the past three decades. For example, using a national sample from the Health and Retirement Study (1998-2004), McLaughlin and colleagues (2010) estimated that no greater than 11.9 percent of adults aged 65 and over aged successfully at four time points of observation. Strawbridge and colleagues (2002) estimated that 18.8 percent were aging successfully in a sample of adults aged 65 and over from Alameda County Study. When using both objective and subjective assessments, Pruchno and colleagues (2010) found that over 80 percent in the sample of a relatively well-educated, younger cohort (50-74) were classified as successful agers. As indicated in a meta-analysis of 28 studies, the prevalence varied greatly from 0.4 to 95 percent, with an average of 35.8 percent of the study subjects being classified as successful agers (Depp \& Jeste, 2009). This considerable variability likely resulted from the sample composition and the different criteria used to operationalize successful aging (Depp \& Jeste, 2009; McLaughlin, Connell, Heeringa, Li, \& Roberts, 2010).

Another line of research documented changes in a single indictor over time (e.g., Andreescu, Chang, Mulsant, \& Ganguli, 2008; Dodge, Du, Sazton, \& Ganguli, 2008; Liang et al., 2008; Thomas, 2012), or examined multiple indicators of successful aging with cross-sectional research designs (e.g., Ng, Broekman, Niti, Gwee, \& Kua, 2009; von 
Faber et al., 2001). These studies suggested older adults maintaining high levels of physical, mental, and social functioning were able to age successfully. The longitudinal studies on a single indicator of successful aging have demonstrated heterogeneity in the aging process (e.g., Andreescu et al., 2008; Dodge et al., 2006; Liang et al., 2008; Thomas, 2012). Few studies, however, have examined multiple aging trajectories based on the multidimensional concept of successful aging. A study by Hsu and Jones (2012) is a notable exception. In a sample of older adults in Taiwan, they identified four aging trajectory groups: successful aging, usual aging, health decline, and care demanding; compared with members in other groups, successful agers had better physical and mental functioning, higher levels of social support, social participation, and economic satisfaction over time (Hsu \& Jones, 2012).

Prior studies also documented gender differences in aging trajectories. Generally, older women have less education achievement and less earned income than older men (McLaughlin et al., 2010; Yang, Hoshi, Wang, Nakayama, \& Kong, 2013). Low socioeconomic status is one of the significant predictors of poor health in old age. Also older women are likely to have more chronic diseases and experience more physical function limitations than older men despite women's longer lives (McLaughlin et al., 2010; Yang et al., 2013). Therefore, women are less likely to age successfully (Pruchno et al., 2010) or may experience different pathways to successful aging. However, findings are inconsistent in the existent literature. Similarly, there is limited evidence that supports socio-demographic profiles of successful aging, that is, higher levels of education, income and wealth, currently marital status, and White ethnicity are related to the likelihood of aging successfully (e.g., Depp \& Jeste, 2009; McLaughlin et al., 2010).

This literature is thus limited by the use of various operational definitions of successful aging, lack of examining changes in multiple aging dimensions, and inconsistent findings. Despite numerous studies generated from Rowe and Kahn's model (e.g., Berkman et al., 1993; McLaughlin et al., 2010), to our knowledge, none of them have simultaneously modeled multiple dimensions of successful aging and examined the temporal changes on these dimensions among older Americans. Further, no studies to date have examined population heterogeneity or the differences in the aging pathways. Therefore, this study aims to (1) identify multiple aging trajectories using six dimensions of physical, mental, cognitive, and social functioning; (2) assess gender differences in aging trajectories; and (3) examine socio-demographic characteristics in relation to aging trajectories.

\section{Methods}

\subsection{Data Source and Study Sample}

Analyses used data from five consecutive waves of the Health and Retirement Study (HRS: 2000, 2002, 2004, 2006, and 2008). The HRS consists of a multistage stratified area probability sample of adults over age 50 living in the contiguous United States (Servais, 2010). Respondents are interviewed every two years, and age-eligible people from younger birth cohorts are added at certain point of time to make the total sample representative of the target population (Servais, 2010). This study used a subset of respondents 65 years of age and older. Proxy respondents were excluded at baseline $(n=2,062)$. Missing cases were imputed for the included samples in the SAS procedure of Proc Traj via maximum likelihood estimation (Nagin, 2005). The final sample size was 9,226, with 3,740 men (40.5\%) and 5,486 women $(59.5 \%)$. Listwise deletion was applied in the multinomial logistic regression analyses, resulting in a total of 9,068 respondents (3,649 men and 5,419 women).

Most variables used in the analyses were retrieved from a special file distributed by the RAND Center for the Study of Aging. The complied data contains cleaned and processed variables with linkage across survey waves (St. Clair et al., 2011). Measures of active engagement were retrieved from five consecutive survey years (2000-2008).

\subsection{Measures}

Guided by Rowe and Kahn's model (1987; 1997) and previous research (e.g., Hsu \& Johns, 2012; McLaughlin et al., 2010), this study used the following six measures to identify successful aging trajectory: (1) chronic diseases, (2) activity of daily living (ADL) disability, (3) physical functioning, (4) depressive symptoms, (5) cognitive functioning, and (6) activity engagement.

Chronic diseases. One of the criteria of successful aging was "no major disease". Thus the diagnoses of five important chronic diseases were assessed, i.e., cancer, chronic lung disease, diabetes, heart disease, and stroke, because these diseases are major causes of death among older adults (McLaughlin et al., 2010). Each disease was scored, and the summary score could range from 0 (no major chronic disease) to 5 (all the chronic diseases).

Disability. Consistent with the MacArthur Studies of Successful aging (Berkman et al., 1993) and previous research (McLaughlin et al., 2010; Strawbridge et al., 2002), this study assessed disability using self-reported difficulties with activities of daily living (ADLs). The assessed activities included walking across a room, dressing, bathing or showering, eating, getting in or out of bed, and using the toilet (McLaughlin et al., 2010). Binary responses (yes/no) were provided to each item. The overall score ranged from 0 (no difficulty) to 6 (difficulties in all six activities). 
Physical functioning. HRS participants were asked whether they had difficulty in performing seven physically demanding tasks, which included walking one block; walking several blocks; climbing one flight of stairs; climbing several flights of stairs; lifting or carrying items weighing more than $10 \mathrm{lbs}$; stooping, kneeling or crouching; and pulling or pushing large objects (St. Clair et al., 2011). Binary responses (yes/no) were summed up to capture functional limitations, with possible ranges from 0 (no difficulty) to 7 (difficulties in all seven tasks).

Depressive symptoms. The eight-item Center for Epidemiologic Studies Depression Scale (CES-D) was used to measure depressive symptoms. This scale was developed for the Established Populations for Epidemiologic Study of the Elderly (Kohout, Berkman, Evans, \& Cornoni-Huntley, 1993), measuring a continuum of psychological distress. The depressive symptom score (range 0-8) was calculated as the number of items endorsed by HRS respondents. Higher scores indicated more psychological distress.

Cognitive functioning. The total cognition summary score was used to indicate individual cognitive functioning; it summarizes the memory and mental status scores. Memory was assessed using two word list recall tasks, i.e., immediate free recall and delayed free recall. Working memory was assessed using the Serial 7's subtraction test. Mental status was measured by the tests of backward counting, date naming, object naming, and President/Vice President naming (St. Clair et al., 2011). The resulting range was 0-35, with higher scores indicating better cognitive functioning.

Activity engagement. In line with previous research (e.g., Bukov, Mass, \& Lampert, 2002; Herzog, Kahn, Morgan, Jackson, \& Antonucci, 1989; Hsu \& Johns, 2012), engagement with life was measured by participation in productive activities, including working for pay, volunteering for organizations, and helping friends, neighbors, or relatives. The summary score of engagement in three activities ranged from 0 to 3 , with higher scores indicating engagement in more activities.

Baseline covariates of aging trajectories. Age was measured by years, ranging from 65 to 104 at baseline (M=74.49, $S D=7.08)$. Race-ethnicity was classified as Hispanic (6\%), non-Hispanic White (82\%), and non-Hispanic Black (12\%). Respondents identifying as other ethnicities were not included in the analyses due to their small sample size $(n=148)$. Education was measured as number of years of completed school, ranging 0-17 $(M=11.83, S D=3.35)$. Household income (range 0 to $\$ 5,539,024)$ was $\log$ transformed $(M=10.14, S D=1.0)$ due to its skewed and wide distribution. Marital status was coded into married (58\%) and non-married (42\%) (see Table 1).

Table 1. Descriptive Statistics of Baseline Characteristics.

\begin{tabular}{lc}
\hline & $M(S D) / N(\%)$ \\
\cline { 2 - 2 } & $74.49(7.08)$ \\
Gender & \\
Male & $3740(41 \%)$ \\
Female & $5486(59 \%)$ \\
Race & \\
Non-Hispanic White (\%) & $7530(82 \%)$ \\
Hispanic (\%) & $1103(12 \%)$ \\
Married (\%) & $593(6 \%)$ \\
Education (years) & $5229(58 \%)$ \\
Household income (log) & $11.83(3.35)$ \\
Chronic diseases & $10.14(1.00)$ \\
Disability & $0.77(0.88)$ \\
Physical functioning & $0.40(1.00)$ \\
Depressive symptoms & $1.96(2.15)$ \\
Cognitive functioning & $1.62(1.91)$ \\
Activity engagement & $21.50(5.35)$ \\
\hline
\end{tabular}

\subsection{Statistical Analysis}

This study applied a multi-trajectory model to estimate multiple aging trajectories over time. The multi-trajectory analysis, based on the group-based trajectory model, is designed to measure the linkage among the trajectories of multiple distinct but related outcomes (Jones \& Nagin, 2007). The basic group-based trajectory model aims to identify groups of individuals with statistically similar progression or trajectories in a single outcome (Nagin, 2005). It assumes 
that the population comprises of a finite mixture of underlying, distinctive developmental trajectories. In the multi-trajectory model, each trajectory group is defined by the multiple trajectories of related outcomes (Nagin, 2005). The size of the trajectory group is measured by the probability of group membership, which can be linked to individual characteristics. The model also computes probabilities that link membership in trajectory groups across outcomes, thus providing a more detailed and varied summary of the developmental connections among multiple outcomes (Nagin, 2005). The likelihood for each individual $i$ given the number of groups $j$ is

$$
P\left(Y_{1}, Y_{2}, \ldots, Y_{K}\right)=\sum_{j} \pi_{j} \prod_{k} f_{k}^{j}\left(Y_{k}\right),
$$

where $k$ is an index of the number of different outcome trajectories in each trajectory group $j\left(k=6\right.$ in this study); $\pi_{\mathrm{j}}$ is the probability of membership in group $j$; and $f(*)$ defines the distribution for each outcome by trajectory group (Jones $\&$ Nagin, 2007). In this study, the aging trajectories were modeled using the Proc Traj Poisson and censored normal options. For the outcomes of chronic diseases, disability, physical functioning, and activity engagement, the Poisson models were used (Jones, Nagin, \& Roeder, 2001):

$$
\log \left(y_{i t}^{i}\right)=\beta_{0}^{j}+\beta_{1}^{j} \text { Time }_{i t}+\beta_{2}^{j} \text { Time }_{i t}^{2}+\beta_{3}^{j} \text { Time }_{i t}^{3}
$$

Depressive symptoms and cognitive functioning were normally distributed, thus the censored normal models were used (Jones et al., 2001):

$$
\left(y_{i t}^{* i}\right)=\beta_{0}^{j}+\beta_{1}^{j} \text { Time }_{i t}+\beta_{2}^{j} \text { Time }_{i t}^{2}+\beta_{3}^{j} t i m e_{i t}^{3}+\varepsilon_{i t}
$$

The basic models provide a predicted shape of each trajectory. In application of these models, iterative testing of the data suggested the need to consider only linear and quadratic specifications of time (also see Zimmer, Martin, Nagin \& Jones, 2012). Separate trajectory models were estimated for men and women. The Proc Traj procedure in SAS program can model up to six outcomes variables simultaneously for multiple trajectories (Hsu \& Jones, 2012). The Bayesian Information Criterion (BIC) is a fit index used to compare competing models with different numbers of trajectories. Theoretical fitness, parsimony principle, and the substantive importance of the groups are also important in determining the optimal trajectory group number (Hsu \& Jones, 2012).

Once the aging trajectory groups were derived from the multi-trajectory model, the group assignment based on posterior probabilities of group membership was used in the multinomial logistic regression analyses to examine the relationship between the aging trajectory groups and the demographic characteristics at baseline in the samples men and women, respectively. One group served as the reference category (successful aging, see below).

\section{Results}

\subsection{Three Aging Trajectories}

Numerous specifications of the models were assessed and compared, including those with quadratic and cubic function of time. As a result, a three-group model was selected and fit the data best in both samples of males and females. Figure 1 displayed the trajectory plots for the three groups in the sample of men. Group $1(38.1 \%$ of the sample, $n=1,423)$ was usual aging group, characterized by the moderate levels of chronic diseases, disability, and physical functional limitation that increased over years, steadily lower level of depressive symptoms, and relatively high levels of cognitive functioning and activity engagement that decreased over time. Group $2(39.6 \%$ of the sample, $n=1,482)$ was successful aging group. Members in this group had few but increasing chronic diseases over time, steadily low levels of disability and physical functional limitation, steadily few depressive symptoms, and high levels of cognitive functioning and activity engagement that decreased over time. Group 3, the pathological aging group, contained $22.3 \%$ of the sample $(n$ = 835). Members in this group exhibited the highest levels of chronic diseases, disability, and physical functional limitation that increased over time, steadily high level of depressive symptoms, and lowest levels of cognitive functioning and activity engagement. Figure 2 displayed the trajectory plots for the three groups in the sample of women. Group 1 (32.7\% of the sample, $n=1,797)$ was classified as successful aging group; Group 2 (38.9\% of the sample, $n=2,143)$ was usual aging group; and Group 3 (28.3\% of the sample, $n=1,546)$ was identified as pathological aging group. The three groups in women exhibited similar characteristics in physical, mental, cognitive, and social functioning as did the sample of men. 

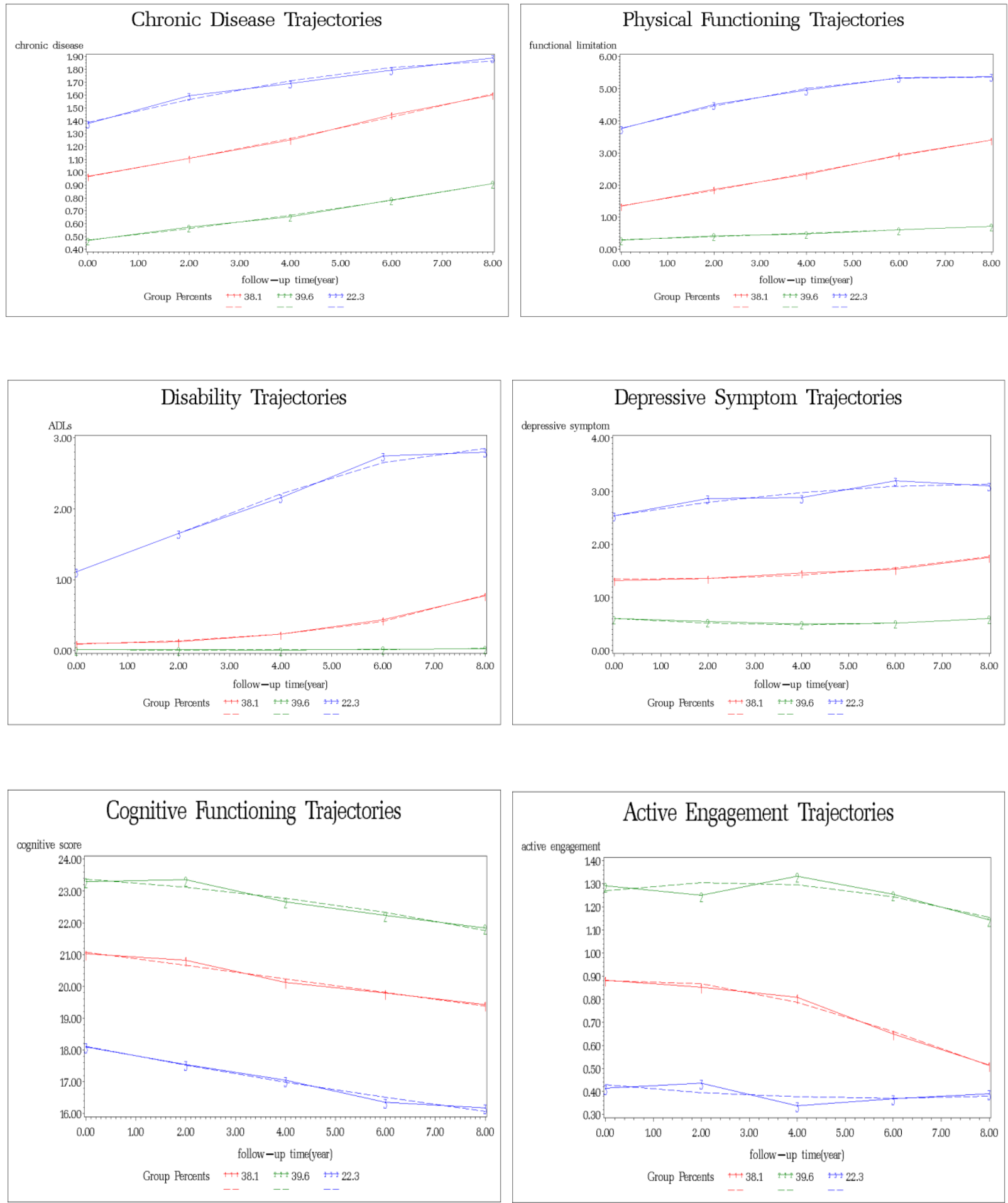

Figure 1. Three aging trajectories for men during 2000-2008. Group 1: Usual aging (38.1\%). Group 2: Successful aging $(39.6 \%)$. Group 3: Diseased aging (22.3\%). Bayesian information criteria: $-131486.5(\mathrm{~N}=3740)$, Akaike information criteria: $-131305.9, \mathrm{~L}=-131247.9$ 

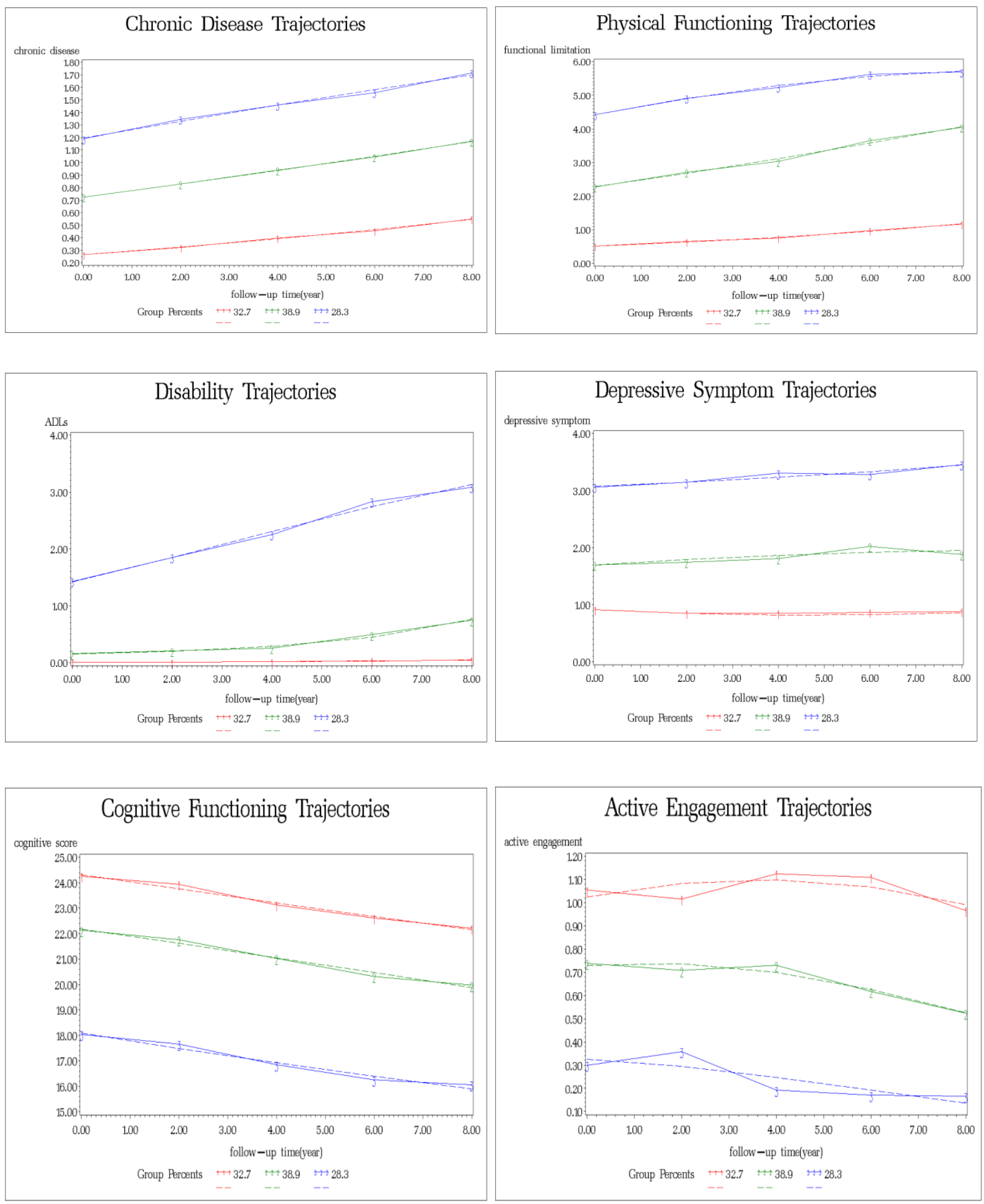

Figure 2. Three aging trajectories for women during 2000-2008. Group 1: Successful aging (32.7\%). Group 2: Usual aging (38.9\%). Group 3 (28.3\%). Bayesian information criteria: -209943.9 ( $\mathrm{N}=5489)$, Akaike information criteria: $-209752.2, \mathrm{~L}=-209694.2$

\subsection{Gender Differences in Aging Trajectories}

Gender differences in group assignments and membership characteristics were examined using chi-square tests and independent $t$-tests. Men were more likely than women to experience successful aging trajectory but less likely to fall into the pathologic aging trajectory $\left[X^{2}(2, N=9,226)=59.7, p<.0001\right]$. Gender differences also existed in the 
membership indicators and characteristics across three groups (Table 2). In general, older women reported fewer diagnosed chronic diseases, but more physical functional limitations and depressive symptoms across three groups. As to ADL disability, women in the successful aging group were not different than their male counterparts at earlier observation periods, but reported more disabilities at later observation periods. In contrast, women in the usual and pathologic aging groups had more disabilities at earlier periods but no gender difference was observed at later times. Women in the successful aging and the usual aging groups usually had higher cognitive functioning scores than male counterparts, but no gender difference was observed in the pathologic aging group. Older men were generally more actively engaged with life across three groups. Women had less household income and were less likely to be married than men across three groups.

Table 2. Gender differences in three aging trajectory groups and membership characteristics.

\begin{tabular}{|c|c|c|c|c|c|c|c|c|c|}
\hline \multirow{2}{*}{$\begin{array}{l}\text { Groups } \\
\text { Gender }\end{array}$} & \multicolumn{3}{|c|}{ Successful aging } & \multicolumn{2}{|c|}{ Usual aging } & & \multicolumn{3}{|c|}{ Pathologic aging } \\
\hline & Men & Women & & Men & Women & & Men & Women & \\
\hline Age (in 2000) & 71.24 & 71.67 & $*$ & 74.71 & 74.80 & & 77.70 & 78.53 & $*$ \\
\hline Non-Hispanic White & 0.87 & 0.85 & & 0.84 & 0.81 & $*$ & 0.75 & 0.74 & \\
\hline Non-Hispanic Black & 0.08 & 0.10 & & 0.10 & 0.12 & & 0.15 & 0.17 & \\
\hline Hispanic & 0.05 & 0.05 & & 0.05 & 0.07 & & 0.09 & 0.09 & \\
\hline Married & 0.84 & 0.57 & $* * *$ & 0.75 & 0.45 & $* * *$ & 0.66 & 0.30 & $* * *$ \\
\hline Education (years) & 13.05 & 12.61 & $* * *$ & 11.82 & 11.81 & & 10.73 & 10.51 & \\
\hline Income $(\log )$ & 10.67 & 10.32 & $* * *$ & 10.33 & 9.98 & $* * *$ & 9.99 & 9.53 & $* * *$ \\
\hline \multicolumn{10}{|l|}{ Chronic diseases } \\
\hline 2000 & 0.46 & 0.26 & $* * *$ & 0.98 & 0.73 & $* * *$ & 1.38 & 1.19 & $* * *$ \\
\hline 2002 & 0.56 & 0.32 & $* * *$ & 1.12 & 0.83 & $* * *$ & 1.59 & 1.35 & $* * *$ \\
\hline 2004 & 0.65 & 0.40 & $* * *$ & 1.26 & 0.94 & $* * *$ & 1.68 & 1.47 & $* * *$ \\
\hline 2006 & 0.78 & 0.46 & $* * *$ & 1.45 & 1.03 & $* * *$ & 1.79 & 1.57 & $* *$ \\
\hline 2008 & 0.91 & 0.55 & $* * *$ & 1.61 & 1.17 & $* * *$ & 1.88 & 1.72 & \\
\hline \multicolumn{10}{|l|}{ Physical functioning } \\
\hline 2000 & 0.28 & 0.50 & $* * *$ & 1.34 & 2.28 & $* * *$ & 3.79 & 4.44 & $* * *$ \\
\hline 2002 & 0.41 & 0.64 & $* * *$ & 1.87 & 2.72 & $* * *$ & 4.52 & 4.92 & $* * *$ \\
\hline 2004 & 0.48 & 0.75 & $* * *$ & 2.31 & 3.03 & $* * *$ & 4.96 & 5.26 & $* *$ \\
\hline 2006 & 0.61 & 0.97 & $* * *$ & 2.94 & 3.68 & $* * *$ & 5.34 & 5.64 & $*$ \\
\hline 2008 & 0.71 & 1.16 & $* * *$ & 3.42 & 4.07 & $* * *$ & 5.38 & 5.70 & $*$ \\
\hline \multicolumn{10}{|l|}{ Disability } \\
\hline 2000 & 0.01 & 0.01 & & 0.09 & 0.15 & $* * *$ & 1.12 & 1.44 & $* * *$ \\
\hline 2002 & 0.01 & 0.01 & & 0.13 & 0.20 & $* * *$ & 1.66 & 1.85 & $*$ \\
\hline 2004 & 0.01 & 0.02 & $* *$ & 0.22 & 0.25 & & 2.16 & 2.27 & \\
\hline 2006 & 0.02 & 0.04 & $*$ & 0.43 & 0.49 & & 2.75 & 2.86 & \\
\hline 2008 & 0.03 & 0.04 & $*$ & 0.77 & 0.75 & & 2.84 & 3.10 & \\
\hline \multicolumn{10}{|l|}{ Depressive symptoms } \\
\hline 2000 & 0.59 & 0.90 & $* * *$ & 1.31 & 1.71 & $* * *$ & 2.57 & 3.07 & $* * *$ \\
\hline 2002 & 0.55 & 0.85 & $* * *$ & 1.35 & 1.75 & $* * *$ & 2.87 & 3.15 & $*$ \\
\hline 2004 & 0.50 & 0.85 & $* * *$ & 1.45 & 1.81 & $* * *$ & 2.88 & 3.30 & $* *$ \\
\hline 2006 & 0.52 & 0.86 & $* * *$ & 1.52 & 2.03 & $* * *$ & 3.21 & 3.30 & \\
\hline 2008 & 0.60 & 0.89 & $* * *$ & 1.76 & 1.88 & & 3.09 & 3.47 & \\
\hline \multicolumn{10}{|l|}{ Cognitive functioning } \\
\hline 2000 & 23.35 & 24.30 & $* * *$ & 20.96 & 22.13 & $* * *$ & 18.11 & 17.98 & \\
\hline 2002 & 23.39 & 23.98 & $* * *$ & 20.79 & 21.73 & $* * *$ & 17.53 & 17.64 & \\
\hline 2004 & 22.67 & 23.13 & $* *$ & 20.11 & 21.01 & $* * *$ & 17.10 & 16.79 & \\
\hline 2006 & 22.26 & 22.63 & $*$ & 19.74 & 20.31 & $* *$ & 16.42 & 16.19 & \\
\hline 2008 & 21.87 & 22.20 & & 19.34 & 19.96 & $*$ & 16.22 & 16.03 & \\
\hline \multicolumn{10}{|l|}{ Activity engagement } \\
\hline 2000 & 1.30 & 1.06 & $* * *$ & 0.87 & 0.74 & $* * *$ & 0.42 & 0.30 & $* * *$ \\
\hline 2002 & 1.25 & 1.02 & $* * *$ & 0.85 & 0.71 & $* * *$ & 0.44 & 0.35 & $* *$ \\
\hline 2004 & 1.34 & 1.12 & $* * *$ & 0.80 & 0.73 & $*$ & 0.34 & 0.19 & $* * *$ \\
\hline 2006 & 1.26 & 1.11 & $* * *$ & 0.65 & 0.62 & & 0.37 & 0.17 & $* * *$ \\
\hline 2008 & 1.15 & 0.97 & $* * *$ & 0.51 & 0.52 & & 0.39 & 0.16 & $* * *$ \\
\hline
\end{tabular}

$* \mathrm{p}<.05, * * \mathrm{p}<.01, * * * \mathrm{p}<.001$ 


\subsection{Socio-demographics of Aging Trajectories}

Multinomial logistic regressions were used to examine the relationship between socio-demographics at baseline and the trajectory groups. The results indicated that the successful aging group was significantly different than the other two groups (Table 3). In both genders, compared with the successful aging group, members in other groups were more likely to be older, less educated, and with less household income. Race/ethnicity and marital status were generally not related to group memberships, except that non-Hispanic Black women were more likely to be in the pathologic aging group than non-Hispanic White women.

Table 3. Results of Multinomial Logistic Regression Analyses.

\begin{tabular}{|c|c|c|c|c|}
\hline \multirow[b]{2}{*}{$\begin{array}{l}\text { Predictors in } \\
\text { baseline }\end{array}$} & \multicolumn{2}{|c|}{ Men } & \multicolumn{2}{|c|}{ Women } \\
\hline & $\begin{array}{l}\text { Usual aging group } \\
(\mathrm{n}=1383)\end{array}$ & $\begin{array}{l}\text { Pathologic aging group } \\
(\mathrm{n}=816)\end{array}$ & $\begin{array}{l}\text { Usual aging group } \\
(\mathrm{n}=2111)\end{array}$ & $\begin{array}{l}\text { Pathologic aging group } \\
(\mathrm{n}=1534)\end{array}$ \\
\hline Age & $1.10 * * *$ & $1.17 * * *$ & $1.08^{* * *}$ & $1.15^{* * *}$ \\
\hline \multicolumn{5}{|l|}{ Non-Hispanic } \\
\hline Black & 0.96 & 1.30 & 1.23 & $1.41 * *$ \\
\hline Hispanic & 0.69 & 1.08 & 0.96 & 0.83 \\
\hline Education & $0.92 * * *$ & $0.89 * * *$ & $0.93 * * *$ & $0.87 * * *$ \\
\hline \multicolumn{5}{|l|}{ Household } \\
\hline Married & 0.87 & 0.83 & 1.05 & 0.98 \\
\hline \multicolumn{3}{|c|}{-2 Log likelihood $=7804 \mathrm{df}=12$} & \multicolumn{2}{|c|}{-2 Log likelihood $=11814 \mathrm{df}=12$} \\
\hline
\end{tabular}

Note: The reference group of the dependent variable was the successful aging group for both men $(\mathrm{n}=1450)$ and women $(\mathrm{n}=1774)$ samples. The reference groups of independent variables were Non-Hispanic White (race-ethnicity), and not married (marital status). $\quad * \mathrm{p}<.05, * * \mathrm{p}<.01, * * * \mathrm{p}<.001$

\section{Discussion}

\subsection{Finding Implications}

Findings of this study provide support for a multidimensional model of successful aging that emphasizes multiple pathways in the aging process, as proposed by Rowe and Kahn (1987; 1997). Through examining temporal changes in six dimensions of physical, mental, cognitive, and social functioning, this study identified three distinct aging trajectories among older Americans. Consistent with the meta-analysis done by Depp and Jeste (2009), the study documented that a sizable proportion (over one third) of older Americans were at low risk of disease and disability, had high physical and cognitive functional capacity, and were actively engaged in society. Differentiated from the successful aging group, about 40 percent of older adults falling in the usual aging group demonstrated certain risk of disease and disability, but were still functioning mentally, cognitively, and socially. In contrast, over one fifth of males and one quarter of females in the study sample were classified into the pathologic aging group. They might have experienced disease-related declines, with least functional capacity in all six domains among the three groups. The distinctions among three aging trajectories indicate population heterogeneity in the aging process, providing a relatively comparative and holistic picture of the aging pathways.

Gender differences exist in the aging trajectories and this study contributes to clarifying previous equivocal findings (e.g., McLaughlin et al., 2010; Newman et al., 2003). Given that older women reported more functional limitations and depressive symptoms as well as lower levels of social engagement, they were less likely than men to age successfully but more likely to fall into the pathologic aging trajectory. Even within the successful aging group, women had lower physical, mental, and social functioning than men. On the contrary, this study documents that women reported fewer diagnosed chronic diseases than men, probably due to the fact that men had higher rates of some chronic diseases (e.g., cancer, coronary and pulmonary disease) (McLaughlin et al., 2010; Murtagh \& Hubert, 2004). However, when examining nonfatal disabling conditions (e.g., fractures, osteoporosis, back problems, osteoarthritis, and depression), old women consistently reported more functional limitations and physical disability, and diminished quality of life than older men (Murtagh \& Hubert, 2004). Gender differences in physical capacity due to primary or comorbid conditions may affect how to define and measure successful aging. Future research need develop gender-specific measures to profile aging trajectories.

Consistent with previous research (e.g., McLaughlin et al., 2010), this study found that younger age and higher levels of education and income were related to the likelihood of aging successfully. Obviously, younger age is related to lower risk of disease and disability or age-related losses. Higher education and income would enable older adults to have great access to health promotion resources, cognitive resources, and psychosocial resources, which in turn help eliminate the adverse effects in later life and increase the likelihood of aging successfully (Beatty, Kamarck, Matthews, \& Shiffman, 
2011; McLaughlin et al., 2010). Also socioeconomic status is related to health-promoting behaviors, such as physical activity, avoidance of tobacco, and nutritious diets during the life course (Pampel, Krueger, \& Denney, 2010). Older adults with healthy behaviors and lifestyle are likely to age successfully.

This study contributes to the literature on successful aging in several ways. Through the use of nationally representative, longitudinal data and the application of the multiple trajectory analysis technique, this study improves our understanding of the multidimensional concept of successful aging and population heterogeneity in the aging process. Although a number of studies were generated in accordance with Rowe and Kahn's model, few of them have incorporated multiple dimensions (i.e., physical, mental, cognitive, and social functioning) of successful aging, and at the same time, examined temporal changes in these dimensions. Besides, most studies assumed population homogeneity in the aging process without investigating the distinction between successful aging and other possible aging trajectories. Static, rigid criteria of successful aging that were used in cross-sectional design studies may mask our understanding of the dynamic nature in the aging process. These criteria may be changeable conditional on chronological age, gender, or other cultural specific contexts. The current study captures the mutable outcomes and classifies older adults with similar trajectory trends in the aging process through the use of multi-trajectory modeling. This dynamic approach helps identify and summarize multi-period aging trajectories, demonstrating individual probabilities or memberships in each identified trajectory. This study also provides strong evidence for gender differences in successful aging and the socioeconomic impacts on the aging trajectory patterns, thus clarifying the mixed findings in the literature. We can never underestimate the salience of social statuses in predicting later life well-being.

\subsection{Limitations}

There are limitations in this study. First, the measures of successful aging used in this study only included objective assessments. As widely debated in the literature, both objective criteria and subjective evaluations are important to assess various aging trajectories (e.g., Pruchno et al., 2010; Strawbridge et al., 2002). The inclusion of subjective components would likely result in different typologies of successful aging trajectory (Pruchno et al., 2010). It is important for future research to incorporate both components to identify and compare different successful aging trajectories.

Second, the six dimensions selected in this study may not be exclusive or valid. For example, although social connection was used to measure activity engagement in previous research (e.g., McLaughlin et al., 2010), the preliminary analysis in this study indicated that it did not distinguish the specific aging trajectories, probably due to the lack of clear conceptualization or valid measures in the HRS. Furthermore, activity engagement was limited to only three types of productive activity: paid work, organizational volunteering, and informal help to friends or relatives. Definitely, other activities such as church attendance, family caregiving, and participation in social clubs or groups would fall within the realm of social engagement and have influences on older adults' health (Thomas, 2012). Further efforts are needed to develop valid measurement of activity engagement and evaluate its role in distinguishing aging trajectories.

Third, transitions among aging trajectories were not examined in this study. It is common that age-relate losses of function lead to transition "from successful to usual, usual to diseased, diseased to impaired" in the aging process (Rowe \& Kahn, 1987, p.148). In addition, extrinsic factors such as life style, diet, exercise and psychosocial factors may affect successful aging trajectories and transitions. Lastly, this analysis may overestimate the overall probability of successful aging, in part due to the lack of consistent criteria across observation periods and in part due to the exclusion of proxy respondents as well as nursing residents.

\subsection{Conclusion}

Despite these limitations, this study contributes to research on successful aging by identifying distinctive aging trajectories, contrasting successful aging with usual and pathologic aging trajectories, and illustrating heterogeneity in the aging process based on within-person functional change and between-person differences in socio-demographic characteristics. Findings provide support for Rowe and Kahn's successful aging model and multiple aging pathways (1987; 1997). Also this study documents gender difference in the aging trajectories and the socioeconomic impacts on the aging trajectory patterns, clarifying equivocal findings in the literature. Future research need improve measurement of successful aging, model transitions among different aging trajectories, and incorporate health behavior and resource variables that have direct effects on the aging outcomes. Given the rapid growth of the older population and the prolonged life expectancy, it is important to promote successful aging through decreasing chronic diseases and the associated disabilities, and improving physical, cognitive, and social functioning in the older population.

\section{Acknowledgements}

This work was supported by the Lois and Samuel Silberman Fund and the Steven Manners Faculty Development Award, University of Pittsburgh Center for Social and Urban Research. 


\section{References}

Andreescu, C., Chang, C. C., Mulsant, B. H., \& Ganguli, M. (2008). Twelve-year depressive symptom trajectories and their predictors in a community sample of older adults. International Psychogeriatrics, 20, 221-236. http://dx.doi.org/10.1017/S1041610207006667

Beatty, D. L., Kamarck, T. W., Matthews, K. A., \& Shiffman, S. (2011). Childhood socioeconomic status is associated with psychosocial resources in African Americans: The Pittsburgh Healthy Heart Project. Health Psychology, 30, 472-480.

Berkman, L. F., Seeman, T. E., Albert, M., Blazer, D., Kahn, R., Mohs, R., Finch, C., Schneider, E., Cotman, C., McClearn, G. et al. (1993). High, usual and impaired functioning in community-dwelling older men and women: Findings from the MacArthur Foundation Research Network on Successful Aging. Journal of Clinical Epidemiology, 46, 1129-1140.

Bukov, A., Mass, I., \& Lampert, T. (2002). Social participation in very old age: Cross-sectional and longitudinal findings from BASE. Journal of Gerontology, Series B: Psychological Sciences and Social Sciences, 57, 510-517. http://dx.doi.org/10.1093/geronb/57.6.P510

Depp, C.A. \& Jeste, D.V. (2009). Definitions and predictors of successful aging: A comprehensive review of larger quantitative studies. Focus: The Journal of Lifelong Learning in Psychiatry, VII, 137-150.

Dodge, H. H., Du, Y., Saxton, J. A., \& Ganguli, M. (2006). Cognitive domains and trajectories of functional independence in nondemented elderly persons. Journal of Gerontology, Series A: Biological Sciences and Medical Sciences, 61, 1330-1337.

Herzog, A. R., Kahn, R. L., Morgan, J. N., Jackson, J. S., \& Antonucci, T. C. (1989). Age differences in productive activities. Journal of Gerontology: Social Sciences, 44, S129-S138. http://dx.doi.org/10.1093/geronj/44.4.S129

Hsu, H.C. \& Jones, B.L. (2012). Multiple trajectories of successful aging of older and younger cohorts. The Gerontologist, 52, 843-856. http://dx.doi.org/10.1093/geron/gns005

Jones, B. L., \& Nagin, D. S. ( 2007). Advances in group-based trajectory modeling and an SAS procedure for estimating them. Sociological Methods and Research, 35, 542-571. http://dx.doi.org/10.1177/0049124106292364

Jones, B. L., Nagin, D. S., \& Roeder, K. (2001). A SAS procedure based on mixture models for estimating developmental trajectories. Sociological Methods and Research, 29, 374-393. http://dx.doi.org/10.1177/0049124101029003005

Kohout, F.J., Berkman, L.F., Evans, D.A., \& Cornoni-Huntley, J. (1993). Two shorter forms of the CES-D (Center for Epidemiological Studies Depression) depression symptoms index. Journal of Aging and Health, 5, 179-193.

Liang, J., Bennett, J. M., Shaw, B. A., Quiñones A. R., Ye, W., Xu, X., et al. (2008). Gender differences in functional status in middle and older age: Are there any age variations? Journal of Gerontology, Series B: Psychological Sciences and Social Sciences, 63, S282-S292.

McLaughlin, S.J., Connell, C.M., Heeringa, S.G., Li, L.W., \& Roberts, L.S. (2010). Successful aging in the United States: Prevalence estimates from a national sample of older adults. Journal of Gerontology: Social Sciences, 65(B), 213-226. http://dx.doi.org/10.1093/geronb/gbp101

Murtagh, K.N. \& Hubert, H.B. (2004). Gender differences in physical disability among an elderly cohort. American Journal of Public Health, 94, 1406-1411.

Nagin, D. S. (2005). Group-based modeling of development. Cambridge, MA: Harvard University Press.

Newman, A.B., Arnold, A.M., Naydeck, B.L. et al. (2003). Successful aging: Effect of subclinical cardiovascular disease. Arch Intern Med., 163, 2315-2311.

Ng, T.P., Broekman, B.F.P., Niti,M., Gwee, X., \& Kua, E.H. (2009). Determinants of successful aging using a multidimensional definition among Chinese elderly in Singapore. The American Journal of Geriatric Psychiatry, 17, 407-416. http://dx.doi.org/10.1097/JGP.0b013e31819a808e

Pampel, F.C., Krueger, P.M., \& Denney, J.T. (2010). Socioeconomic disparities in health behaviors. Annual Review of Sociology, 36, 349-370.

Pruchno, R. A., Wilson-Genderson, M., Rose, M., \& Cartwright, F. (2010). Successful aging: Early influences and contemporary characteristics. The Gerontologist, 50, 821-833. http://dx.doi.org/10.1093/geront/gnq041

Rowe, J. W., \& Kahn, R. L. (1987). Human aging: Usual and successful. Science, 237, 143-149.

Rowe, J. W., \& Kahn, R. L. (1997). Successful aging. The Gerontologist, 37, 433-440. 
Servais, M. A. (2010). Overview of HRS public data files for cross-sectional and longitudinal analysis. Ann Arbor: Survey Research Center, Institute for Social Research, University of Michigan. Retrieved from http://hrsonline.isr.umich.edu/sitedocs/dmgt/OverviewofHRSPublicData.pdf.

St. Clair, P., Bugliari, D., Campbell, N., Chien, S., Hayden, O., Hurd, M. et al. (2011). RAND HRS data documentation, version L. Labor \& Population Program, RAND Center for the Study of Aging. Retrieved from www.rand.org/content/dam/rand/www/external/labor/aging/dataprod/randhrsL.pdf.

Strawbridge, W. J., Wallhagen, M. I., \& Cohen, R. D. (2002). Successful aging and well-being: Self-rated compared with Rowe and Kahn. The Gerontologist, 42, 727-733.

Thomas, P.A. (2012). Trajectories of social engagement and mortality in late life. Journal of Aging and Health, 24, 547-568. http://dx.doi.org/10.1177/0898264311432310

Von Faber M, Bootsma-van der Wiel A, van Exel E, Gussekloo L, Lagaay AM, van Dongen E, et al. (2001). Successful aging in the oldest old: Who can be characterized as successfully aged? Archives of Internal Medicine, 161, 26942700 .

Yang, S., Hoshi, T., Wang, S., Nakayama, N., \& Kong, F. (2013). Socioeconomic Status, Comorbidity, Activity Limitation, and Healthy Life Expectancy in Older Men and Women: A 6-Year Follow-Up Study in Japan. Journal of Applied Gerontology. http://dx.doi.org/10.1177/0733464813503041

Zimmer, Z., Martin, L.G., Nagin, D.S., \& Jones, B.L. (2012). Modeling disability trajectories and mortality of the oldest-old in China. Demography, 49, 291-314.

\section{(cc) $\mathrm{BY}$}

This work is licensed under a Creative Commons Attribution 3.0 License. 